\title{
Immersive Authoring of Virtual Reality Training
}

\author{
F Cassola* \\ INESC TEC, FEUP
}

\author{
M Pinto ${ }^{\dagger}$ \\ INESC TEC
}

\author{
D Mendes \\ INESC TEC, FEUP
}

\author{
L Morgado§ \\ INESC TEC, UAb
}

\author{
A Coelholl \\ INESC TEC, FEUP
}

H Paredes"
INESC TEC, UTAD

\begin{abstract}
The use of VR in industrial training contributes to reduce costs and risks, supporting more frequent and diversified use of experiential learning activities, an approach with proven results. In this work, we present an innovative immersive authoring tool for experiential learning in VR-based training. It enables a trainer to structure an entire VR training course in an immersive environment, defining its sub-components, models, tools, and settings, as well as specifying by demonstration the actions to be performed by trainees. The trainees performing the immersive training course have their actions recorded and matched to the ones specified by the trainer.
\end{abstract}

Index Terms: Applied computing-Education-Interactive learning environments; Computing methodologies-Computer graphicsGraphics systems and interfaces_-Virtual reality

\section{INTRODUCTION}

In the industrial sector, the ability to adapt to requests with a high degree of customization and complexity implies longer cycle times. The application of virtual reality (VR) is a promising approach [1], helping operators minimize errors and to increase safety. Virtual reality environments are also used to perform operational tasks in industrial settings, as an experiential and situated learning approach, which has shown good results in areas such as work safety [2], medicine [3], mechanical maintenance [4] and mining [5]. Indeed, the use of a virtual environment to carry out learning activities presents a wide range of advantages when compared to training in a real context, namely reducing costs and risks $[6,7]$, higher control of training procedures (for example, scheduling training sessions and identifying action complexity), promoting experiential and situated learning, the ability to ensure the replication of the simulations carried out by trainees, the empowerment of self-learning processes $[8,9]$, and triggering emotions furthering the interest and engagement of learners [10].

However, the creation of training experiences in VR is limited, due to its expensive and time-consuming development process (e.g. Unity or Unreal Engine) that requires software development experts [11]. To mitigate this hurdle, authoring tools have been proposed for development of VR applications for training and certification (e.g. EON Reality and XVR Simulation). Current limitations of this approaches include: lack of integration with existing data: such tools do not allow the creation of VR scenarios from pre-existing data in organizations, such as engineering CAD files; desktop-oriented, non-immersive authoring processes that detach the immersive experience from its creation (users specify training procedures without experiencing the trainee task performance perspective).

\footnotetext{
*e-mail: fjcm@inesctec.pt

†e-mail: manuel.a.alves@inesctec.pt

†e-mail: danielmendes@fe.up.pt

$\S$ e-mail: leonel.morgado@inesctec.pt

Ile-mail: antonio.coelho@inesctec.pt

"e-mail: hugo.paredes@inesctec.pt
}
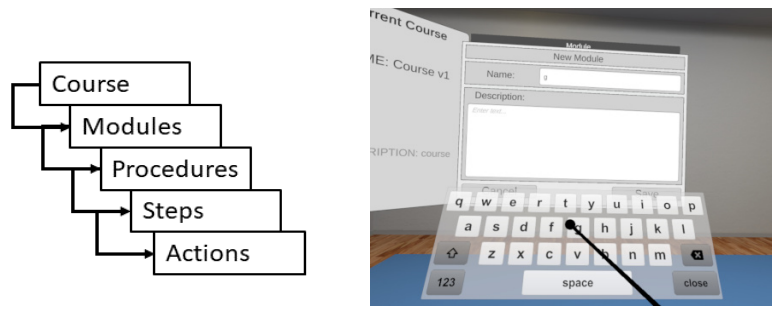

Figure 1: Left: Course structure; Right: Module creation panels.

We tackle these limitations with a VR authoring tool, enabling immersive authoring of immersive training. With an authentic VR environment from pre-existing CAD data (created by the Vestas Wind Systems Company team), digital representations are generated, following the concept of digital twins [12]. This authenticity brings trainers and trainees experience varied and rich experiential in learning for industrial maintenance scenarios.

\section{VR AUthoring TOOL}

We developed an immersive authoring tool in VR for trainers to create immersive training courses. The actions that the trainee can perform in the virtual environment are executed by example on industrial machinery, and descriptive information is added. These actions are structured as procedures, as described in the next subsection The tool was designed as a generic approach training procedures on engineering components, and is currently being applied with training of maintenance procedures on wind turbines.

Defining Course Structure In the VR environment, the trainer can create courses and specify their elements: training modules and their procedures, and the steps of each procedure (Fig. 1 Left). This information defines the context for the subsequent immersive actions, both for their creation and their editing. This is currently being done in a traditional windowed dialogue metaphor (Fig. 1 Right), only with the side-by-side information at a lateral pane, to support head-rotation viewing. We envision recreating this task in a more immersive mode of interaction.

Setting Up the Environment The tool allows the trainer to setup the training environment, specifying: the visual space for the training (Fig. 2 Left); the starting position of the trainee (Fig. 2

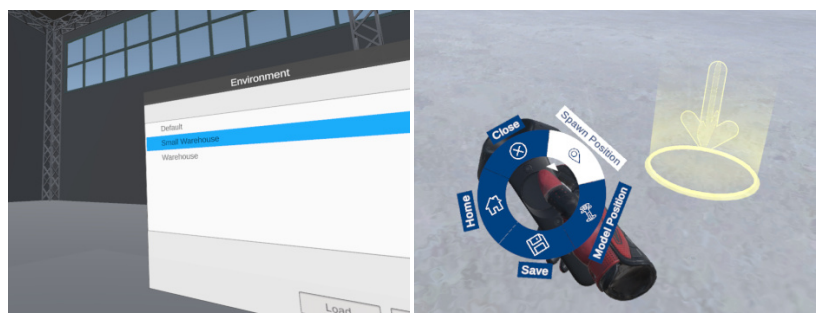

Figure 2: Left: Choosing an environment; Right: Defining the initial position for the trainee. 


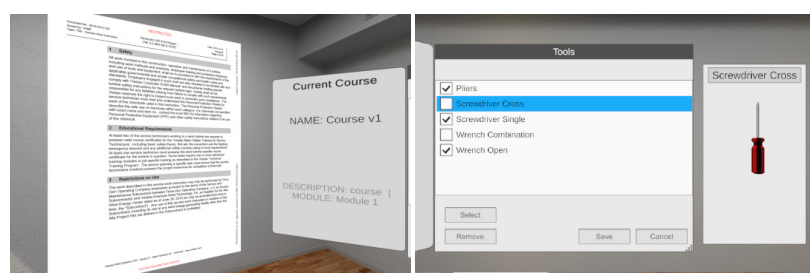

Figure 3: Left: Instructions' document; Right: Choosing tools.

Right); the technical documentation for the procedures (Fig. 3 Left); the tools to be used during the training (Fig. 3 Right); the 3D model of the machinery upon which the procedures will be executed (Fig. 4 Left). With the 3D model of the machinery already visible, the trainer can also restrict interactions with it to a subset of parts (Fig. 4 Right). This may be desired to simplify the complexity of executing a specific procedure.
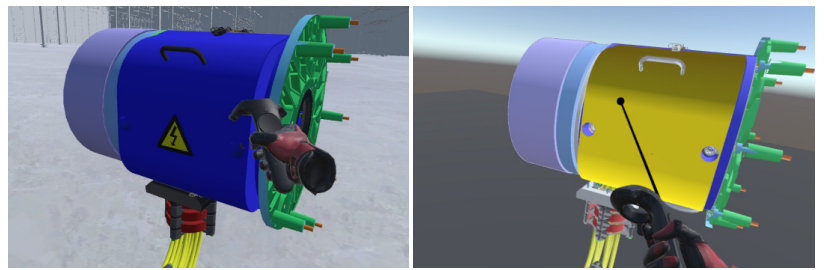

Figure 4: Left: Positioning the target training component model on the environment; Right: Defining the interactive parts of the model.

Procedure Recording After structuring the course and setting it up, the trainer can demonstrate the actions to be performed by the trainee. Firstly, a specific step in the course structure is selected (Fig. 5 Left). Then, the trainer performs the desired actions directly upon the 3D model (Fig. 5 Right). The system will record these as a virtual choreography for that step, at a semantic level associated with the training intent, using XAPI syntax, rather than low-level software behaviours [13]. For instance, removing a machine cover is stored as "remove cover" rather than the sequence of hovering with the cursor over a polygon, picking it up, moving it outside the model, and releasing it away from an attachment point.

Training Execution Once the trainer recorded all course procedures, a trainee can execute the training. Trainees can watch a replay of the trainer's actions, and/or attempt to perform the procedures. First, the trainee chooses the appropriate tools among those set up by the trainer. Then, with the set-up technical documentation available within the immersive environment via menu option, procedure execution upon the 3D model can start. Only the parts set up for interaction can be interacted upon. All trainee actions are recorded by the system monitors. As for the trainer actions, these are recorded as a virtual choreography, at a semantic level associated with the

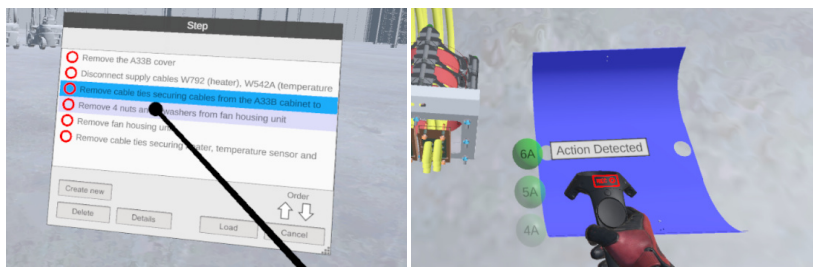

Figure 5: Left: Choosing step to record; Right: Recording actions by demonstration. training. Thus, they can be analysed by the trainer subsequently, to the point of full replay, if necessary, for learning analysis perspective. This also enables automated matching of the trainee's actions with the trainer's intent, since irrelevant actions can be disregarded: the trainee is provided feedback about the correctly-performed actions.

\section{Conclusion}

This tool serves as a proof-of-concept that demonstrated the feasibility of immersive authoring of immersive training. The industrial machinery (wind turbines) is particularly detailed and originated various challenges, such as heavy model loading, and massive numbers of parts to interact with. The system thus is an exemplar of interaction solutions that can be employed in other systems, enabling other research inquiries to be explored in the future.

\section{ACKNOWLEDGMENTS}

Work financed by the ERDF - European Regional Development Fund through the Operational Programme for Competitiveness and Internationalisation - COMPETE 2020 under the Portugal 2020 Partnership Agreement, and through the Portuguese National Innovation Agency (ANI) as a part of project POCI-01-0247-FEDER-038524.

\section{REFERENCES}

[1] Å. Fast-Berglund, T. Fässberg, F. Hellman, A. Davidsson, and J. Stahre, "Relations between complexity, quality and cognitive automation in mixed-model assembly," Journal of Manufacturing Systems, vol. 32, no. $3,2013$.

[2] J. Bertram, J. Moskaliuk, and U. Cress, "Virtual training: Making reality work?," Computers in Human Behavior, vol. 43, 2015.

[3] N. E. Seymour, A. G. Gallagher, S. A. Roman, M. K. O’brien, V. K. Bansal, D. K. Andersen, and R. M. Satava, "Virtual reality training improves operating room performance: results of a randomized, doubleblinded study," Annals of surgery, vol. 236, no. 4, 2002.

[4] A. Pinheiro, P. Fernandes, A. Maia, G. Cruz, D. Pedrosa, B. Fonseca, H. Paredes, P. Martins, L. Morgado, and J. Rafael, "Development of a mechanical maintenance training simulator in opensimulator for f-16 aircraft engines," Entertainment Computing, vol. 5, no. 4, 2014

[5] A. Grabowski and J. Jankowski, "Virtual reality-based pilot training for underground coal miners," Safety science, vol. 72, 2015.

[6] K. K. Bhagat, W.-K. Liou, and C.-Y. Chang, "A cost-effective interactive $3 \mathrm{~d}$ virtual reality system applied to military live firing training,' Virtual Reality, vol. 20, no. 2, 2016.

[7] A. A. García, I. G. Bobadilla, G. A. Figueroa, and M. P. Ramírez, "Virtual reality training system for maintenance and operation of highvoltage overhead power lines," Virtual Reality, vol. 20, no. 1, 2016.

[8] H. De Visser, M. O. Watson, O. Salvado, and J. D. Passenger, "Progress in virtual reality simulators for surgical training and certification," Medical journal of Australia, vol. 194, 2011.

[9] A. Mossel, A. Peer, J. Göllner, and H. Kaufmann, "Requirements analysis on a virtual reality training system for cbrn crisis preparedness," in Proceedings of the 59th Annual Meeting of the ISSS, vol. 1, 2015.

[10] L. M. Alves Fernandes, G. Cruz Matos, D. Azevedo, R. Rodrigues Nunes, H. Paredes, L. Morgado, L. F. Barbosa, P. Martins, B. Fonseca, P. Cristóvão, et al., "Exploring educational immersive videogames: an empirical study with a $3 \mathrm{~d}$ multimodal interaction prototype," Behaviour \& Information Technology, vol. 35, no. 11, 2016.

[11] H. Gaspar, L. Morgado, H. Mamede, T. Oliveira, B. Manjón, and C. Gütl, "Research priorities in immersive learning technology: the perspectives of the ilrn community," Virtual Reality, 2019.

[12] F. Tao, J. Cheng, Q. Qi, M. Zhang, H. Zhang, and F. Sui, "Digital twin-driven product design, manufacturing and service with big data," The International Journal of Advanced Manufacturing Technology, vol. 94, no. 9-12, 2018.

[13] D. Lacet, F. Penicheiro, L. Morgado, and A. Coelho, "Preserving story choreographies across multiple platforms: An approach to platformindependent reuse of characters' behaviors for games, simulations, animations and interactive videos," in Proceedings of the 9th International Conference on Digital and Interactive Arts, 2019. 